\title{
Nuclear Charge Radii of the Nickel Isotopes ${ }^{58-68,70} \mathrm{Ni}$
}

S. Malbrunot-Ettenauer, ${ }^{1, *}$ S. Kaufmann, ${ }^{2, \dagger}$ S. Bacca $\odot,{ }^{3,4}$ C. Barbieri $\odot,{ }^{5,6,7}$ J. Billowes,${ }^{8}$ M. L. Bissell, ${ }^{8}$ K. Blaum $\odot,{ }^{9}$ B. Cheal, ${ }^{10}$ T. Duguet, ${ }^{11,12}$ R. F. Garcia Ruiz, ${ }^{8,1, *}$ W. Gins, ${ }^{12,8}$ C. Gorges, ${ }^{2}$ G. Hagen $\odot,{ }^{13,14}$ H. Heylen, ${ }^{9,1}$ J. D. Holt $\odot,{ }^{15,16}$ G. R. Jansen $\odot,{ }^{13,17}$ A. Kanellakopoulos, ${ }^{12, \|}$ M. Kortelainen $\odot{ }^{18}$ T. Miyagi $1{ }^{15}$ P. Navrátilø ${ }^{15}$ W. Nazarewicz ${ }^{19},{ }^{19}$ R. Neugart, ${ }^{9,20}$ G. Neyens, ${ }^{1,12}$ W. Nörtershäuser ${ }^{2,},{ }^{2,}$ S. J. Novario, ${ }^{14,13}$ T. Papenbrock $\odot,{ }^{14,13}$ T. Ratajczyk, ${ }^{2}$ P.-G. Reinhard, ${ }^{21}$ L. V. Rodríguez, ${ }^{1,9,22}$ R. Sánchez $\odot,{ }^{23}$ S. Sailer, ${ }^{24}$ A. Schwenk $\odot,{ }^{2,25,9}$ J. Simonis, ${ }^{3}$ V. Somà, ${ }^{11}$ S. R. Stroberg, ${ }^{26}$ L. Wehner ${ }^{20}$ C. Wraith, ${ }^{10}$ L. Xie, ${ }^{8}$ Z. Y. Xu, ${ }^{12}$ X. F. Yang $\odot,{ }^{27,12}$ and D. T. Yordanov ${ }^{22}$ ${ }^{1}$ Experimental Physics Department, CERN, CH-1211 Geneva 23, Switzerland

${ }^{2}$ Institut für Kernphysik, Technische Universität Darmstadt, D-64289 Darmstadt, Germany

${ }^{3}$ Institut für Kernphysik and PRISMA ${ }^{+}$Cluster of Excellence, Johannes Gutenberg-Universität Mainz, D-55128 Mainz, Germany

${ }^{4}$ Helmholtz-Institut Mainz, GSI Helmholtzzentrum für Schwerionenforschung GmbH, D-64291 Darmstadt, Germany

${ }^{5}$ Department of Physics, University of Surrey, Guildford, GU2 7XH, United Kingdom

${ }^{6}$ Dipartimento di Fisica, Università degli Studi di Milano, Via Celoria 16, 20133 Milano, Italy ${ }^{7}$ INFN, Sezione di Milano, Via Celoria 16, 20133 Milano, Italy

${ }^{8}$ School of Physics and Astronomy, The University of Manchester, Manchester M13 9PL, United Kingdom

${ }^{9}$ Max-Planck-Institut für Kernphysik, D-69117 Heidelberg, Germany

${ }^{10}$ Oliver Lodge Laboratory, University of Liverpool, Oxford Street, Liverpool L69 7ZE, United Kingdom

${ }^{11}$ IRFU, CEA, Université Paris-Saclay, 91191 Gif-sur-Yvette, France

${ }^{12}$ KU Leuven, Instituut voor Kern- en Stralingsfysica, B-3001 Leuven, Belgium

${ }^{13}$ Physics Division, Oak Ridge National Laboratory, Oak Ridge, Tennessee 37831, USA

${ }^{14}$ Department of Physics and Astronomy, University of Tennessee, Knoxville, Tennessee 37996, USA

${ }^{15}$ TRIUMF 4004 Wesbrook Mall, Vancouver, British Columbia V6T 2A3, Canada

${ }^{16}$ Department of Physics, McGill University, Montréal, Quebec H3A 2T8, Canada

${ }^{17}$ National Center for Computational Sciences, Oak Ridge National Laboratory, Oak Ridge, Tennessee 37831, USA

${ }^{18}$ Department of Physics, University of Jyväskylä, P.O. Box 35 (YFL), FI-40014 University of Jyväskylä, Finland

${ }^{19}$ Department of Physics and Astronomy and FRIB Laboratory, Michigan State University, East Lansing, Michigan 48824, USA

${ }^{20}$ Institut für Kernchemie, Johannes Gutenberg-Universität Mainz, D-55128 Mainz, Germany

${ }^{21}$ Institut für Theoretische Physik II, Universität Erlangen-Nürnberg, 91058 Erlangen, Germany

${ }^{22}$ Institut de Physique Nucléaire, CNRS-IN2P3, Université Paris-Sud, Université Paris-Saclay, 91406 Orsay, France

${ }^{23}$ GSI Helmholtzzentrum für Schwerionenforschung GmbH, D-64291 Darmstadt, Germany

${ }^{24}$ Technische Universität München, D-80333 München, Germany

${ }^{25}$ ExtreMe Matter Institute EMMI, GSI Helmholtzzentrum für Schwerionenforschung GmbH, D-64291 Darmstadt, Germany

${ }^{26}$ Department of Physics, University of Washington, Seattle, Washington, D.C. 98195, USA

${ }^{27}$ School of Physics and State Key Laboratory of Nuclear Physics and Technology, Peking University, Beijing 100871, China

(Received 18 June 2021; revised 5 October 2021; accepted 22 October 2021; published 14 January 2022)

Collinear laser spectroscopy is performed on the nickel isotopes ${ }^{58-68,70} \mathrm{Ni}$, using a time-resolved photon counting system. From the measured isotope shifts, nuclear charge radii $R_{c}$ are extracted and compared to theoretical results. Three $a b$ initio approaches all employ, among others, the chiral interaction $\mathrm{NNLO}_{\text {sat }}$, which allows an assessment of their accuracy. We find agreement with experiment in differential radii $\delta\left\langle r_{c}^{2}\right\rangle$ for all employed $a b$ initio methods and interactions, while the absolute radii are consistent with data only for $\mathrm{NNLO}_{\text {sat }}$. Within nuclear density functional theory, the Skyrme functional SV-min matches experiment more closely than the Fayans functional $\mathrm{Fy}(\Delta r, \mathrm{HFB})$.

DOI: 10.1103/PhysRevLett.128.022502

Published by the American Physical Society under the terms of the Creative Commons Attribution 4.0 International license. Further distribution of this work must maintain attribution to the author(s) and the published article's title, journal citation, and DOI.
Introduction.-The accurate description of rich physics phenomena encountered in atomic nuclei remains a formidable challenge for contemporary nuclear theory. The long-term goal of nuclear physics is thus to develop a universal framework to consistently describe atomic nuclei across the entire nuclear chart. Research in recent years has led to remarkable advances in nuclear many-body methods 
[1-7] as well as in the development of nuclear forces based on chiral effective field theory (EFT), rooted in symmetries of QCD and based on pion exchanges and short-ranged interactions [8-10]. A significant theoretical effort has been dedicated to the description of electromagnetic properties such as nuclear charge radii $R_{c}$. Since charge radii can be measured with high accuracy, they serve as robust benchmarks for nuclear theory. Presently, the region of mediumto heavy-mass nuclei constitutes the testing ground for developing the coherent theoretical nuclear framework. An important element of this endeavor is to connect ab initio models to nuclear density functional theory (DFT). In addition to $a b$ initio calculations, well-calibrated energy density functionals, such as the Fayans functional, are capable of a successful description of nuclear charge radii for multiple isotopic chains ranging from potassium $(Z=19)$ all the way to tin $(Z=50)$ [11-17].

In this Letter, we report nuclear charge radii of nickel isotopes ( $\mathrm{Ni}, Z=28$ ) which, in terms of $R_{c}$, constitutes the last unexplored "magic" isotopic chain in this mass region. While the charge radius of ${ }^{68} \mathrm{Ni}$ was reported earlier [18], we here present additionally the results for ${ }^{59,63,65-67,70} \mathrm{Ni}$. The experimental data are compared with two DFT approaches as well as three independent $a b$ initio methods based on chiral EFT interactions.

Experiment.-The experiment at ISOLDE/CERN has been described previously in [18]. Details on the general setup can be found in [19]. In brief, $\mathrm{Ni}$ isotopes were produced in a uranium carbide target bombarded with proton pulses of $1.4-\mathrm{GeV}$ energy. Ions were formed by resonant laser ionization with RILIS [20] and accelerated in a first and a second beamtime to about 30 and $40 \mathrm{keV}$, respectively. Different ISOLDE targets were used with the aim to increase production and to suppress isobars, but they behaved comparably. After mass selection in a highresolution mass separator, the ions were injected into the radio-frequency quadrupole (RFQ) ion beam cooler and buncher ISCOOL [21] where they were accumulated for typically $10-100 \mathrm{~ms}$. After extraction as a short ion bunch, the ions were transported to the collinear laser spectroscopy beam line COLLAPS, where the beam was superimposed with a copropagating laser beam. Bunching reduces the otherwise dominant background of scattered laser light compared to a continuous beam [22]. The ion beam energy was determined by the high-voltage applied to ISCOOL, which was recorded by a precision high-voltage divider. In the first beamtime, a $30-\mathrm{kV}$ divider was available, while a 50-kV divider was provided by PTB Braunschweig later on. This allowed independent voltage calibrations and the use of a higher beam energy, favorable for laser-spectroscopic resolution.

Laser spectroscopy on the neutral $\mathrm{Ni}$ atoms was performed after neutralization of the ions in a charge-exchange cell $[23,24]$ filled with potassium vapor. A frequencydoubled single-mode $\mathrm{cw}$ titanium-sapphire laser stabilized with a high-resolution wavemeter [25,26] was used to excite the $3 d^{9} 4 s^{3} D_{3} \rightarrow 3 d^{9} 4 p^{3} P_{2}$ transition at $352.45 \mathrm{~nm}$. The wavemeter was calibrated regularly with a stabilized helium-neon laser. Fluorescence photons from spontaneous emission were detected by four photomultiplier tubes. All isotopes were measured alternating with the reference isotope ${ }^{60} \mathrm{Ni}$ to compensate for remaining long-term drifts in ion velocity or laser frequency.

For the present Letter, a new data acquisition system called "TILDA" [27] was employed for the first time at COLLAPS. It is based on photon tagging with reference to ISCOOL's release trigger [28] and relaxes the need for hard-wired gates set during a beamtime. Comparable schemes have previously been employed at other laser experiments with bunched ion beams [29-33]. A typical spectrum recorded with TILDA is shown for ${ }^{65} \mathrm{Ni}$ in Fig. 1(a): The $x$ axis represents the laser frequency calculated from the scanning voltage at the chargeexchange cell, while the $y$ axis is the time elapsed since the RFQ extraction pulse was recorded. The color represents the number of photons detected within a 100-ns interval during 900 extractions from the RFQ. The time structure of the ion bunch is shown in Fig. 1(b), where the counts at a specified time are integrated over all frequencies. Similarly, summing all counts at a fixed frequency within the (adjustable) time interval between 53 and $57 \mu$ s reveals the resonance spectrum of the isotope in Fig. 1(c).

According to an analysis with ISOLTRAP's multireflection time-of-flight mass spectrometer [34], the beam of the most exotic isotope ${ }^{70} \mathrm{Ni}$ was dominated by the isobar ${ }^{70} \mathrm{Ga}$ with a ratio of of $\approx 1: 10^{4}$. The large amount of isobaric ions can cause an overfilling of ISCOOL and a corresponding shift in beam energy due to the ions' spacecharge potential, which can degrade the accuracy of the

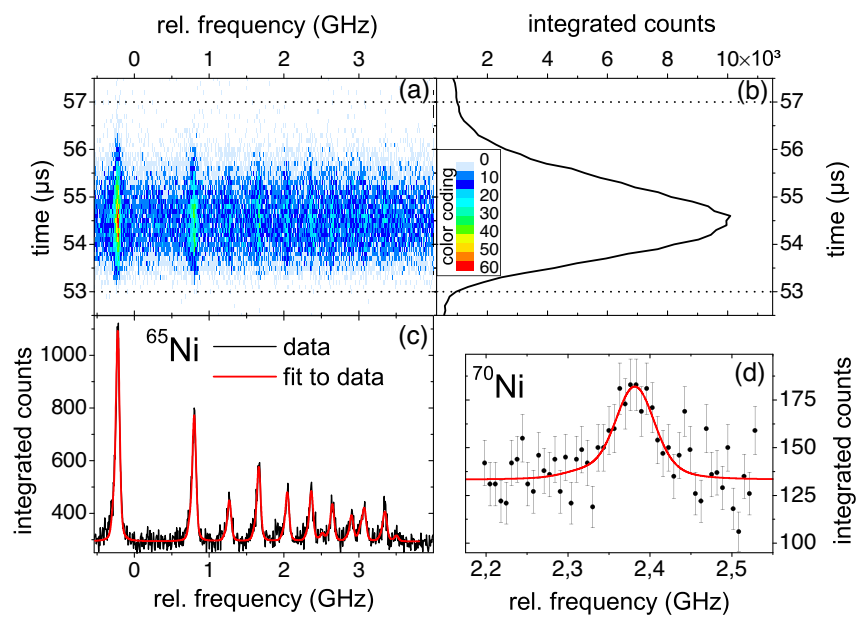

FIG. 1. Frequency-time spectrum of (a) a ${ }^{65} \mathrm{Ni}$ resonance, (b) the temporal ion-bunch structure, and (c) the laserspectroscopic resonance spectrum. (d) A resonance of ${ }^{70} \mathrm{Ni}$. See text for details. 
spectroscopic measurements. Moreover, nonresonant light emitted by the unwanted ions after collisional excitation or neutralization in the charge-exchange cell will reduce the sensitivity for ${ }^{70} \mathrm{Ni}$. To suppress ${ }^{70} \mathrm{Ga}$, we took advantage of the different target-release properties of the two elements: the beam gate at ISOLDE, allowing the ions to be transported to the experiments, stayed closed during the first $2 \mathrm{~s}$ after the proton impact. Then, most of the more volatile ${ }^{70} \mathrm{Ga}$ had been released from the target while the remaining fraction of ${ }^{70} \mathrm{Ni}\left(T_{1 / 2}=6 \mathrm{~s}\right)$ was accumulated for $1.2 \mathrm{~s}$ in the RFQ and then sent as a single bunch to COLLAPS, before the next proton pulse arrived. A ${ }^{70} \mathrm{Ni}$ resonance is shown in Fig. 1(d).

Analysis.-Isotope shifts $\delta \nu^{60, A}=\nu^{A}-\nu^{60}$ for all isotopes were calculated from their respective center frequency $\nu^{A}$ with respect to the center frequency $\nu^{60}$ of the reference isotope ${ }^{60} \mathrm{Ni}$. Both beamtimes were analyzed individually and a linear displacement in their isotope shifts was corrected by introducing a correction to the main acceleration voltage within the uncertainty of the corresponding voltage dividers. The main acceleration voltage of $30 \mathrm{kV}$ (first beamtime) was reduced by $3.5 \mathrm{~V}$, and the $40 \mathrm{kV}$ (second beamtime) was increased by $2.5 \mathrm{~V}$ in the analysis. A still remaining scatter in the isotope shifts of individual isotopes as obtained in the two beamtimes could not be explained by their statistical uncertainties. However, this variation was not systematic and could not be traced back to definite reasons individually. Therefore, an additional statistical uncertainty was added to all isotopes, such that the scatter appeared statistically reasonable, i.e., the $\chi_{\text {red }}^{2}$ calculated from the deviations between the final isotope shifts of the two beamtimes and their average was reduced to 1 . Results are listed in Table I. The changes in mean-square nuclear charge radii $\delta\left\langle r_{c}^{2}\right\rangle^{60, A} \equiv$ $\left\langle r_{c}^{2}\right\rangle^{A}-\left\langle r_{c}^{2}\right\rangle^{60}$ are obtained using the field-shift factor $F=-783(94) \mathrm{MHz} / \mathrm{fm}^{2}$ and the mass-shift factor $M_{\alpha=396}=950(5) \mathrm{GHzu}$, as explained in [18]. These values are in excellent agreement with independent measurements reported in [35]. Negligible deviations from our values in [18] arise from a correction in the analysis code but lead only to insignificant changes of $\delta\left\langle r_{c}^{2}\right\rangle$ values. The uncertainties of $\delta\left\langle r_{c}^{2}\right\rangle^{60, A}$ are dominated by the correlated error based on the uncertainty of $F$. The absolute charge radii $R_{c} \equiv\left\langle r_{c}^{2}\right\rangle^{1 / 2}$ are obtained from $\delta\left\langle r_{c}^{2}\right\rangle^{60, A}$ by utilizing $R_{c}\left({ }^{60} \mathrm{Ni}\right)=3.806(2)$ fm [36].

Theory.-Ab initio approaches compute the mean-square charge radius $\left\langle r_{c}^{2}\right\rangle$ starting from the calculated point-proton mean-square radius $\left\langle r_{p}^{2}\right\rangle$,

$$
\left\langle r_{c}^{2}\right\rangle=\left\langle r_{p}^{2}\right\rangle+\left\langle R_{p}^{2}\right\rangle+\frac{N}{Z}\left\langle R_{n}^{2}\right\rangle+\left\langle r^{2}\right\rangle_{\mathrm{so}}+\frac{3 \hbar^{2}}{4 m_{p}^{2} c^{2}},
$$

where $\left\langle R_{p}^{2}\right\rangle$ and $\left\langle R_{n}^{2}\right\rangle$ are the mean-square charge radii of the proton and the neutron, respectively, $\left\langle r^{2}\right\rangle_{\text {so }}$ denotes a
TABLE I. Measured isotope shifts $\delta \nu^{60, A}$ relative to ${ }^{60} \mathrm{Ni}$ with statistical uncertainties in parentheses and systematic uncertainties in square brackets. Values for the stable isotopes and ${ }^{68} \mathrm{Ni}$ are those from [18]. The statistical uncertainty includes variations between the two beamtimes that are partially of systematic but uncorrelated origin and change statistically from isotope to isotope, while the systematic uncertainty is restricted to the correlated uncertainty caused by the high-voltage measurement. The extracted change in mean-square charge radius $\delta\left\langle r_{c}^{2}\right\rangle^{60, A}$ and the total charge radii $R_{c}$ are listed with the total uncertainties. Please note that there are diminutive corrections (flips in the last digit) in $\delta\left\langle r_{c}^{2}\right\rangle^{60, A}$ compared to [18] caused by a correction in the analysis code.

\begin{tabular}{cccc}
\hline \hline$A$ & $\delta \nu^{60, A} / \mathrm{MHz}$ & $\delta\left\langle r_{c}^{2}\right\rangle^{60, A} / \mathrm{fm}^{2}$ & $R_{c} / \mathrm{fm}$ \\
\hline 58 & $-509.1(25)[42]$ & $-0.275(8)$ & $3.770(2)$ \\
59 & $-214.3(27)[22]$ & $-0.180(9)$ & $3.782(2)$ \\
60 & 0.0 & 0.0 & $3.806(2)$ \\
61 & $280.8(27)[20]$ & $0.082(5)$ & $3.817(2)$ \\
62 & $503.9(25)[39]$ & $0.223(5)$ & $3.835(2)$ \\
63 & $784.9(26)[27]$ & $0.277(8)$ & $3.842(2)$ \\
64 & $1027.2(25)[77]$ & $0.367(10)$ & $3.854(2)$ \\
65 & $1317.5(26)[94]$ & $0.385(18)$ & $3.856(3)$ \\
66 & $1526.8(26)[113]$ & $0.493(17)$ & $3.870(3)$ \\
67 & $1796.6(26)[130]$ & $0.514(25)$ & $3.873(3)$ \\
68 & $1992.3(27)[147]$ & $0.619(24)$ & $3.886(3)$ \\
70 & $2377.2(49)[181]$ & $0.806(24)$ & $3.910(3)$ \\
\hline \hline
\end{tabular}

spin-orbit correction [1,37], and the last term corresponds to the relativistic Darwin-Foldy correction [38], with $m_{p}$ being the proton mass [39]. The intrinsic (i.e., with respect to the center of mass) squared charge radius operator $[1,40,41]$ is employed for $\left\langle r_{p}^{2}\right\rangle$ in all calculations. In the present Letter, the values of $\left\langle R_{p}^{2}\right\rangle=0.709 \mathrm{fm}^{2}[42,43]$ and $\left\langle R_{n}^{2}\right\rangle=-0.106 \mathrm{fm}^{2}$ [44] were used.

We employ the following two- plus three-nucleon $(3 N)$ interactions from chiral EFT: (i) $\mathrm{NNLO}_{\text {sat }}$ [45], which gives a good description of charge radii in light- and mid-mass isotopes but somewhat underbinds finite nuclei $[1,2,7,12,15,46,47]$, (ii) $1.8 / 2.0(\mathrm{EM})[6,48,49]$, and (iii) $N N+3 N(\operatorname{lnl})$ [46], which reproduce ground-state and excitation energies throughout the medium- and heavymass region, but generally underpredict absolute charge radii $[6,7,50]$. The present Letter addresses a long sequence of charge radii along the $\mathrm{Ni}$ isotopic chain for the first time with three $a b$ initio techniques, using these three nuclear interactions. This provides a new, stringent accuracy benchmark of state-of-the-art methods, which implement different computational schemes. Importantly, a thorough evaluation of theoretical uncertainties is carried out for each many-body technique, as briefly described in the following.

The self-consistent Green's function (SCGF) approach [51-53] is a full-space correlation-expansion method applicable to the description of medium-mass nuclei 
[7,46,54-58]. Nickel isotopes were recently addressed in Ref. [46], where the calculation of radii, however, was not optimized and lacked theoretical uncertainties. Here, we present nickel charge radii with a full analysis of basis convergence and an assessment of associated theoretical errors. To this end, SCGF calculations are performed in the second-order Gorkov algebraic diagrammatic construction $[\mathrm{ADC}(2)]$ scheme $[59,60]$ using a spherical harmonic-oscillator basis including up to 14 major shells $\left[e_{\max } \equiv \max (2 n+l)=13\right]$, with matrix elements of threebody operators further restricted to $e_{3 \max }=16$. Theoretical errors comprise uncertainties arising from both many-body and model-space truncations. The former are estimated from differences between $\operatorname{ADC}(2)$ and $\operatorname{ADC}(3)$ [61,62] calculations, available for closed-shell isotopes. The latter are evaluated from a range of oscillator frequencies $\hbar \Omega$ within $2 \mathrm{MeV}$ from the optimal values.

The valence-space in-medium similarity renormalization group (VS-IMSRG) method [63-69] decouples a valencespace Hamiltonian and consistent operators from the fullspace problem via an approximate unitary transformation. To obtain charge radii, we first decouple the core and valence-space intrinsic proton mean-squared radius operator and then apply Eq. (1). We use the IMSRG(2) approximation where induced operators are truncated at the two-body level and the ensemble normal ordering procedure [67,70], which captures the physics of $3 N$ forces between valence particles. We take the neutron $p_{3 / 2}, p_{1 / 2}$, $f_{5 / 2}, g_{9 / 2}$ valence space outside a ${ }^{56} \mathrm{Ni}$ core, decouple a valence-space Hamiltonian for each isotope studied and diagonalize with the KSHELL code [71] to obtain expectation values for the intrinsic proton mean-square radius operator. While model-space uncertainties are obtained analogously to SCGF, errors due to the many-body method cannot be estimated currently [72]. The $e_{\max } / e_{3 \max }$ and basis choices are as in Ref. [6].

The coupled-cluster method performs a similarity transformation of the Hamiltonian and decouples a reference state from its $n$-particle- $n$-hole $(n p-n h)$ excitations [73-76]. This method was used to compute the structure of doubly magic nuclei and their neighbors $[2,3,18,77-79]$ and can also be extended to open-shell nuclei [80]. Our calculations for nickel isotopes employ a single-particle basis of up to 13 harmonic-oscillator shells with a frequency $\hbar \Omega=16 \mathrm{MeV}$; matrix elements of three-nucleon forces are truncated at $e_{3 \max }=16$. We start from an axially symmetric Hartree-Fock reference, normal order the resulting Hamiltonian with respect to this state, and truncate it at the two-body level [81,82]. The ensuing calculations employ the coupled cluster single-double approximation, i.e., $1 p-1 h$ and $2 p-2 h$ excitations of the reference are fully decoupled. While this captures (only) about $90 \%$ of the correlation energy, the omission of $3 p-3 h$ excitations has a much smaller effect on radii and introduces an estimated $1 \%$ uncertainty. Uncertainties from the finite model space are estimated from the difference between calculations in 11 and 13 harmonic-oscillator shells. Overall, we estimate coupled-cluster uncertainties on $R_{c}$ to be $+2 \% /-1 \%$.

The fourth theory considered is nuclear DFT [83]. Here, we focus on nonrelativistic energy density functionals (EDFs) and employ two EDF parametrizations, namely SV-min [84] as representative of the widely used Skyrme functionals and $\mathrm{Fy}(\Delta r, \mathrm{HFB})$ as the recent example of a Fayans functional [13]. Both have the basic structure in common and are calibrated with the same fitting strategy to the same large body of nuclear ground-state data (energy, radii, surface thickness,...) as described in [84]. The Fayans functional $\mathrm{Fy}(\Delta r, \mathrm{HFB})$ differs in that it contains additional gradient terms in surface and pairing energies $[85,86]$ and that isotopic shifts of charge radii in the calcium chain were added to the optimization dataset. The rms charge radii are computed directly from the nuclear charge form factor. The latter is obtained from folding the proton and neutron densities with the intrinsic charge and magnetic densities of the nucleons (for details, see Ref. [87]). The calculations are done with codes allowing for deformed ground states, for SV-min with SkyAx [88] and for Fy $(\Delta r, \mathrm{HFB})$ with a version of HFBTHO [89] extended to Fayans EDF. Results for spherical nuclei have been counterchecked with our spherical BCS/HFB code, the one that was used for the calibration of both functionals $[13,84]$. DFT parametrizations carry statistical uncertainties [84] as well as systematic errors related to principle limitations of the model [90].

Discussion.-Theoretical and experimental nuclear charge radii are compared in Fig. 2. Charge radii $R_{c}$ provide a comparison on the absolute scale, while the differential charge radii $\delta\left\langle r_{c}^{2}\right\rangle$ probe local variations in the nuclear charge distribution more closely, since various theoretical uncertainties cancel in $\delta\left\langle r_{c}^{2}\right\rangle$. For instance, the errors on $R_{c}$ in DFT contain a sizable, nearly constant offset along the chain reflecting a certain vibrational softness for all $\mathrm{Ni}$ isotopes. These vibrational corrections enhance total radii and are thus predominantly positive, but are greatly reduced in $\delta\left\langle r_{c}^{2}\right\rangle$.

For SV-min based DFT as well as for all $a b$ initio calculations based on $\mathrm{NNLO}_{\text {sat }}$, the overall agreement with experiment is very good. For both $R_{c}$ and $\delta\left\langle r_{c}^{2}\right\rangle$, the experimental values are within, or very close to, the theoretical error band, which is on the order of $\approx 1 \%$. The same holds for the differential radii $\delta\left\langle r_{c}^{2}\right\rangle$ when considering $a b$ initio results for the other employed nuclear interactions, see Fig. 2(d), while those deviate notably from experiment in the absolute charge radii $R_{c}$, as shown in Fig. 2(c). This is in line with the expectation from previous work $[7,15]$.

Within the same nuclear many-body method, calculations of $R_{c}$ with $\mathrm{NNLO}_{\text {sat }}$ disagree with the results of both $1.8 / 2.0(\mathrm{EM})$ and $N N+3 N(\operatorname{lnl})$. This illustrates the sensitivity of $R_{c}$ on the accurate encoding of the relevant physics for medium-mass nuclei into nuclear 


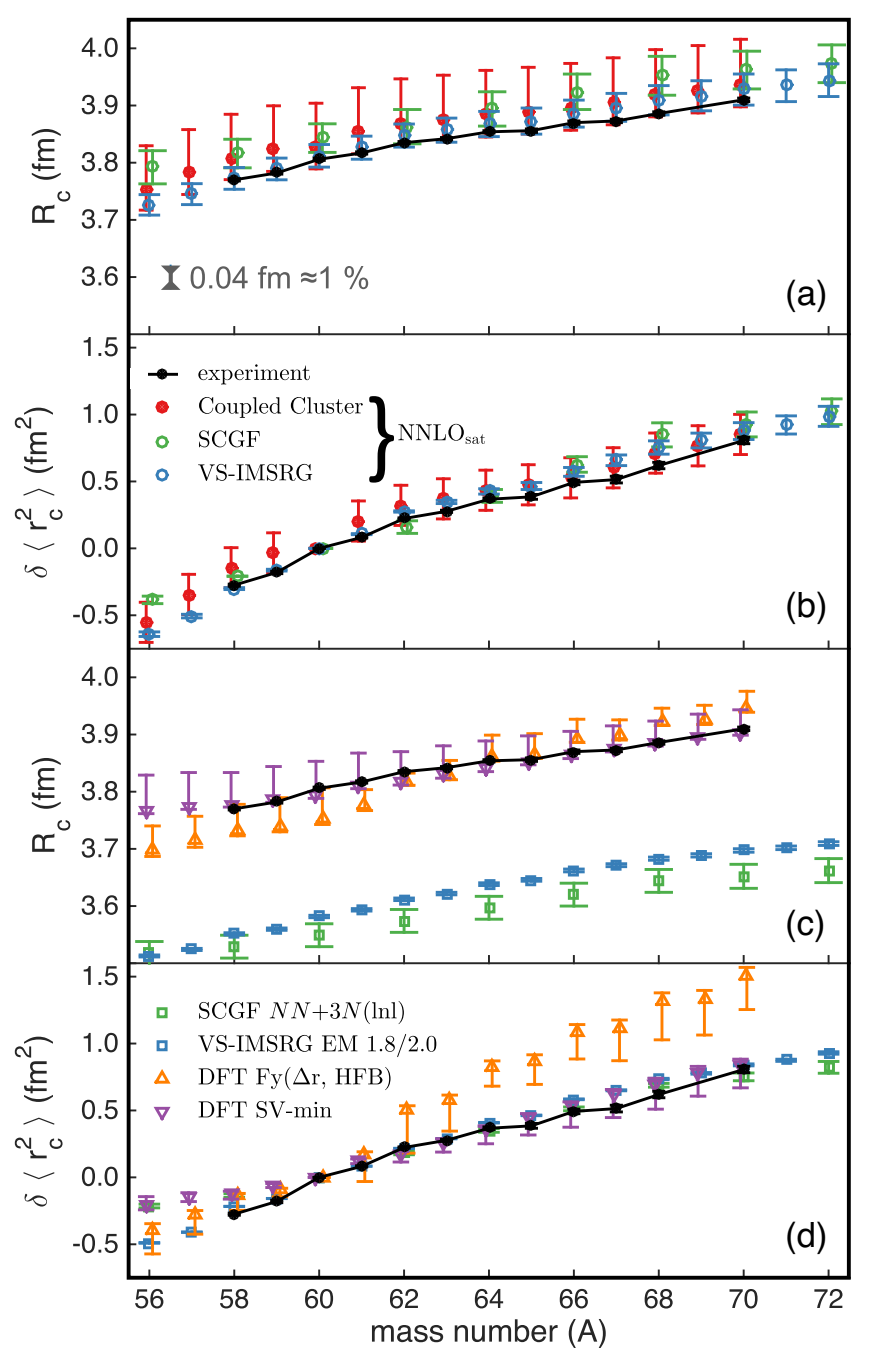

FIG. 2. Nuclear charge radii $R_{c}$ (a,c) and differentials $\delta\left\langle r_{c}^{2}\right\rangle^{60, A}$ (b,d) of $\mathrm{Ni}$ isotopes with respect to ${ }^{60} \mathrm{Ni}$ as reference. Experimental data are compared to theoretical results. See text for details.

forces $[45,49]$. On the other hand, a comprehensive assessment of uncertainties due to a many-body method itself remains a challenge. Employing the same nuclear interaction in conjunction with different many-body methods is one way to evaluate many-body uncertainties. As shown in Figs. 2(a) and 2(b), the results of SCGF, VS-IMSRG, and coupled-cluster theory, all utilizing $\mathrm{NNLO}_{\text {sat }}$, agree with each other within the theoretical uncertainties, thus providing strong evidence for the accuracy of the methods. Small differences can be seen for ${ }^{56} \mathrm{Ni}$, where uncertainties of SCGF and VS-IMSRG do not overlap. Note that the error bars in VS-IMSRG account for model-space uncertainties only. We have confirmed that the latter are consistent in size across different methods.

With respect to nuclear charge radii, the Fayans functional has been very successful in describing an odd-even staggering as well as characteristic kinks typically found at shell closures $[12,13,15-17]$. In contrast, DFT utilizing Skyrme functionals such as SV-min generally fails to reproduce both. However, compared to the large odd-even staggering in $\mathrm{Ca}[12,13]$ or the sizeable kink at $N=82$ in $\mathrm{Sn}$ [17], charge radii along the measured $\mathrm{Ni}$ isotopes do not exhibit these features very prominently. Interestingly, the $\mathrm{SV}$-min follows in this case the experimental trend more closely compared to Fy $(\Delta r$, HFB), see Fig. 2(d). On closer inspection, analogous conclusions for the midshell region also hold for the charge radii of $\mathrm{Cu}$ [15] and $\mathrm{Sn}$ isotopes [17]. A potential deficiency of the present Fayans functional could be its lack of an isovector component in its pairing part [91]. Hence, future efforts in Fayans-based DFT will focus on pinning down the (presently unused) isovector term in the pairing functional, see Ref. [92].

Summary.-Collinear laser spectroscopy of short-lived nickel isotopes ${ }^{58-68,70} \mathrm{Ni}$ was performed. The extracted nuclear mean-square charge radii $R_{c}$ benchmark theoretical work applying density functional theory as well as three $a b$ initio methods. When the same chiral EFT-based nuclear potential $\mathrm{NNLO}_{\text {sat }}$ is utilized in all $a b$ initio calculations, their results show excellent consistency and they agree well with experiment. Calculations exploiting other nuclear interactions perform equally well for $\delta\left\langle r_{c}^{2}\right\rangle$, but struggle in reproducing the absolute radii. Interestingly, in the absence of prominent features such as unusually large odd-even staggering or kinks in $R_{c}$, which have been successfully described by Fayans-based functionals, Skyrme-based DFT yields results closer to experiment. Overall, this comparative work combining experiment, density functional theory, and $a b$ initio calculations establishes a theoretical accuracy of $\sim 1 \%$ for the description of nuclear charge radii in the Ni region.

We acknowledge the support of the ISOLDE Collaboration and technical teams, the ISOLTRAP group, and funding from the European Union's Horizon 2020 program under Grant Agreement No. 654002. We thank the Physikalisch Technische Bundesanstalt (PTB) Braunschweig for the loan of a precision high-voltage divider. This work was supported by the Max-Planck Society, the Deutsche Forschungsgemeinschaft (DFG, German Research Foundation)—ProjectId 279384907-SFB 1245, the Collaborative Research Center [The Low-Energy Frontier of the Standard Model (SFB 1044)], the Cluster of Excellence "Precision Physics, Fundamental Interactions, and Structure of Matter" (PRISMA $^{+}$EXC 2118/1) funded by DFG within the German Excellence Strategy—Projektnummer 39083149, the BMBF under Contracts No. 05P18RDCIA, No. 05P18RDFN1, and No. 05P19RDFN1, the FWO (Belgium), GOA 15/010 from KU Leuven, NSERC, and the Office of Nuclear Physics, U.S. Department of Energy, under Awards No. DE-FG02-96ER40963, No. DESC0013365, No. DE-SC0018083, and No. DESC0018223 (NUCLEI SciDAC-4 Collaboration). Computer time was provided by the Innovative and Novel Computational Impact 
on Theory and Experiment (INCITE) program. This research used resources of the Oak Ridge Leadership Computing Facility located at Oak Ridge National Laboratory, which is supported by the Office of Science of the Department of Energy under Award No. DE-AC05-00OR22725. The calculations presented in this work were also performed on "Mogon II" at Johannes Gutenberg-Universität in Mainz. Computational resources for DFT calculations were partly provided by the CSC-IT Center for Science, Ltd. (Finland). SCGF calculations were performed by using HPC resources from GENCI-TGCC, France (Contract No. A007057392) and at the DiRAC Complexity system at the University of Leicester, UK (BIS National E-infrastructure capital Grant No. ST/K000373/1 and STFC Grant No. ST/K0003259/1). This work was also supported by consolidated grants from STFC (UK)—ST/L005516/1, ST/L005670/1, ST/L005794/ 1, ST/P004423/1, and ST/P004598/1. TRIUMF receives federal funding via a contribution agreement with the National Research Council of Canada. This work profited from R\&D carried out in the frame of the FAIR Phase-0 program of LASPEC/ NUSTAR in which TILDA was developed.

*stephan.ettenauer@cern.ch

${ }^{\dagger}$ Present address: Institut für Kernchemie, Johannes Gutenberg-Universität Mainz, D-55128 Mainz, Germany.

*Present address: Massachusetts Institute of Technology, Cambridge, Massachusetts, USA.

${ }^{\S}$ Present address: Department of Physics, University of Jyväskylä, P.O. Box 35 (YFL), FI-40014 Jyväskylä, Finland.

"Present address: HEPIA Geneva, HES-SO, 1202 Geneva, Switzerland.

"wnoertershaeuser@ikp.tu-darmstadt.de

[1] G. Hagen, A. Ekström, C. Forssén, G. R. Jansen, W. Nazarewicz, T. Papenbrock, K. A. Wendt, S. Bacca, N. Barnea, B. Carlsson, C. Drischler, K. Hebeler, M. HjorthJensen, M. Miorelli, G. Orlandini, A. Schwenk, and J. Simonis, Nat. Phys. 12, 186 (2016).

[2] T. D. Morris, J. Simonis, S. R. Stroberg, C. Stumpf, G. Hagen, J. D. Holt, G. R. Jansen, T. Papenbrock, R. Roth, and A. Schwenk, Phys. Rev. Lett. 120, 152503 (2018).

[3] P. Gysbers, G. Hagen, J. D. Holt, G. R. Jansen, T. D. Morris, P. Navrátil, T. Papenbrock, S. Quaglioni, A. Schwenk, S. R. Stroberg, and K. A. Wendt, Nat. Phys. 15, 428 (2019).

[4] H. Hergert, Front. Phys. 8, 379 (2020).

[5] P. Arthuis, C. Barbieri, M. Vorabbi, and P. Finelli, Phys. Rev. Lett. 125, 182501 (2020).

[6] S. R. Stroberg, J. D. Holt, A. Schwenk, and J. Simonis, Phys. Rev. Lett. 126, 022501 (2021).

[7] V. Somà, C. Barbieri, T. Duguet, and P. Navrátil, Eur. Phys. J. A 57, 135 (2021).

[8] E. Epelbaum, H.-W. Hammer, and Ulf-G. Meißner, Rev. Mod. Phys. 81, 1773 (2009).

[9] R. Machleidt and D. Entem, Phys. Rep. 503, 1 (2011).

[10] H.-W. Hammer, S. König, and U. van Kolck, Rev. Mod. Phys. 92, 025004 (2020).
[11] Á. Koszorús et al., Nat. Phys. 17, 439 (2021).

[12] R. F. Garcia Ruiz et al., Nat. Phys. 12, 594 (2016).

[13] A. J. Miller, K. Minamisono, A. Klose, D. Garand, C. Kujawa, J. D. Lantis, Y. Liu, B. Maß, P. F. Mantica, W. Nazarewicz, W. Nörtershäuser, S. V. Pineda, P. G. Reinhard, D. M. Rossi, F. Sommer, C. Sumithrarachchi, A. Teigelhöfer, and J. Watkins, Nat. Phys. 15, 432 (2019).

[14] K. Minamisono, D. M. Rossi, R. Beerwerth, S. Fritzsche, D. Garand, A. Klose, Y. Liu, B. Maßß, P. F. Mantica, A. J. Miller, P. Müller, W. Nazarewicz, W. Nörtershäuser, E. Olsen, M. R. Pearson, P.-G. Reinhard, E. E. Saperstein, C. Sumithrarachchi, and S. V. Tolokonnikov, Phys. Rev. Lett. 117, 252501 (2016).

[15] R. P. de Groote et al., Nat. Phys. 16, 620 (2020).

[16] M. Hammen et al., Phys. Rev. Lett. 121, 102501 (2018).

[17] C. Gorges et al., Phys. Rev. Lett. 122, 192502 (2019).

[18] S. Kaufmann et al., Phys. Rev. Lett. 124, 132502 (2020).

[19] R. Neugart, J. Billowes, M. L. Bissell, K. Blaum, B. Cheal, K. T. Flanagan, G. Neyens, W. Nörtershäuser, and D. T. Yordanov, J. Phys. G 44, 064002 (2017).

[20] B. A. Marsh, Rev. Sci. Instrum. 85, $02 B 923$ (2014).

[21] H. Frånberg, P. Delahaye, J. Billowes, K. Blaum, R. Catherall, F. Duval, O. Gianfrancesco, T. Giles, A. Jokinen, M. Lindroos, D. Lunney, E. Mane, and I. Podadera, Nucl. Instrum. Methods Phys. Res., Sect. B 266, 4502 (2008).

[22] A. Nieminen, P. Campbell, J. Billowes, D. H. Forest, J. A. R. Griffith, J. Huikari, A. Jokinen, I. D. Moore, R. Moore, G. Tungate, and J. Äystö, Phys. Rev. Lett. 88, 094801 (2002).

[23] A. Mueller, F. Buchinger, W. Klempt, E. Otten, R. Neugart, C. Ekström, and J. Heinemeier, Nucl. Phys. A403, 234 (1983).

[24] A. Klose, K. Minamisono, C. Geppert, N. Frömmgen, M. Hammen, J. Krämer, A. Krieger, C. Levy, P. Mantica, W. Nörtershäuser, and S. Vinnikova, Nucl. Instrum. Methods Phys. Res., Sect. A 678, 114 (2012).

[25] M. Verlinde, K. Dockx, S. Geldhof, K. König, D. Studer, T. E. Cocolios, R. P. de Groote, R. Ferrer, Y. Kudryavtsev, T. Kieck, I. Moore, W. Nörtershäuser, S. Raeder, P. van den Bergh, P. van Duppen, and K. Wendt, Appl. Phys. B 126, 85 (2020).

[26] K. König, P. Imgram, J. Krämer, B. Maaß, K. Mohr, T. Ratajczyk, F. Sommer, and W. Nörtershäuser, Appl. Phys. B 126, 86 (2020).

[27] S. Kaufmann, T. Beyer, K. Blaum, M. Block, E. D. Ch, K. Eberhardt, M. Eibach, C. Geppert, C. Gorges, J. Grund, M. Hammen, J. Krämer, N. Sz, W. Nörtershäuser, D. Renisch, F. Schneider, and K. Wendt, J. Phys. Conf. Ser. 599, 012033 (2015).

[28] A. Kanellakopoulos et al., Phys. Rev. C 102, 054331 (2020).

[29] A. Voss, M. R. Pearson, J. Billowes, F. Buchinger, B. Cheal, J. E. Crawford, A. A. Kwiatkowski, C. D. Philip Levy, and O. Shelbaya, Phys. Rev. Lett. 111, 122501 (2013).

[30] M. Lochmann et al., Phys. Rev. A 90, 030501(R) (2014).

[31] D. M. Rossi, K. Minamisono, B. R. Barquest, G. Bollen, K. Cooper, M. Davis, K. Hammerton, M. Hughes, P. F. Mantica, D. J. Morrissey, R. Ringle, J. A. Rodriguez, C. A. Ryder, S. Schwarz, R. Strum, C. Sumithrarachchi, D. Tarazona, and S. Zhao, Rev. Sci. Instrum. 85, 093503 (2014).

[32] R. F. Garcia Ruiz, A. R. Vernon, C. L. Binnersley, B. K. Sahoo, M. Bissell, J. Billowes, T. E. Cocolios, W. Gins, R. P. 
de Groote, K. T. Flanagan, A. Koszorus, K. M. Lynch, G. Neyens, C. M. Ricketts, K. D. A. Wendt, S. G. Wilkins, and X. F. Yang, Phys. Rev. X 8, 041005 (2018).

[33] R. de Groote, A. de Roubin, P. Campbell, B. Cheal, C. Devlin, T. Eronen, S. Geldhof, I. Moore, M. Reponen, S. Rinta-Antila, and M. Schuh, Nucl. Instrum. Methods Phys. Res., Sect. B 463, 437 (2020).

[34] R. Wolf, F. Wienholtz, D. Atanasov, D. Beck, K. Blaum, C. Borgmann, F. Herfurth, M. Kowalska, S. Kreim, Y. A. Litvinov, D. Lunney, V. Manea, D. Neidherr, M. Rosenbusch, L. Schweikhard, J. Stanja, and K. Zuber, Int. J. Mass Spectrom. 349-350, 123 (2013).

[35] K. König, F. Sommer, J. Lantis, K. Minamisono, W. Nörtershäuser, S. Pineda, and R. Powel, Phys. Rev. C 103, 054305 (2021).

[36] G. Fricke and K. Heilig, Landolt-Börnstein, Group I: Elementary Particles, Nuclei and Atoms (Springer, Berlin, Heidelberg, New York, 2004), Vol. 20.

[37] C. J. Horowitz and J. Piekarewicz, Phys. Rev. C 86, 045503 (2012).

[38] J. L. Friar, J. Martorell, and D. W. L. Sprung, Phys. Rev. A 56, 4579 (1997).

[39] F. Heiße, F. Köhler-Langes, S. Rau, J. Hou, S. Junck, A. Kracke, A. Mooser, W. Quint, S. Ulmer, G. Werth, K. Blaum, and S. Sturm, Phys. Rev. Lett. 119, 033001 (2017).

[40] G. Hagen, T. Papenbrock, D. J. Dean, and M. Hjorth-Jensen, Phys. Rev. C 82, 034330 (2010).

[41] A. Cipollone, C. Barbieri, and P. Navrátil, Phys. Rev. C 92, 014306 (2015).

[42] R. Pohl et al., Nature (London) 466, 213 (2010).

[43] W. Xiong et al., Nature (London) 575, 147 (2019).

[44] A. A. Filin, V. Baru, E. Epelbaum, H. Krebs, D. Möller, and P. Reinert, Phys. Rev. Lett. 124, 082501 (2020).

[45] A. Ekström, G. R. Jansen, K. A. Wendt, G. Hagen, T. Papenbrock, B. D. Carlsson, C. Forssén, M. Hjorth-Jensen, P. Navrátil, and W. Nazarewicz, Phys. Rev. C 91, 051301(R) (2015).

[46] V. Somà, P. Navrátil, F. Raimondi, C. Barbieri, and T. Duguet, Phys. Rev. C 101, 014318 (2020).

[47] H. Heylen et al., Phys. Rev. C 103, 014318 (2021).

[48] K. Hebeler, S. K. Bogner, R. J. Furnstahl, A. Nogga, and A. Schwenk, Phys. Rev. C 83, 031301(R) (2011).

[49] J. Simonis, S. R. Stroberg, K. Hebeler, J. D. Holt, and A. Schwenk, Phys. Rev. C 96, 014303 (2017).

[50] T. Miyagi, S. R. Stroberg, P. Navrátil, K. Hebeler, and J. D. Holt, Phys. Rev. C 105, 014302 (2022).

[51] W. H. Dickhoff and C. Barbieri, Prog. Part. Nucl. Phys. 52, 377 (2004).

[52] A. Carbone, A. Cipollone, C. Barbieri, A. Rios, and A. Polls, Phys. Rev. C 88, 054326 (2013).

[53] V. Somà, Front. Phys. 8, 340 (2020).

[54] F. Raimondi and C. Barbieri, Phys. Rev. C 99, 054327 (2019).

[55] C. Barbieri, N. Rocco, and V. Somà, Phys. Rev. C 100, 062501(R) (2019).

[56] A. Idini, C. Barbieri, and P. Navrátil, Phys. Rev. Lett. 123, 092501 (2019).

[57] S. Chen et al., Phys. Rev. Lett. 123, 142501 (2019).

[58] Y. Sun et al., Phys. Lett. B 802, 135215 (2020).
[59] V. Somà, T. Duguet, and C. Barbieri, Phys. Rev. C 84, 064317 (2011).

[60] V. Somà, C. Barbieri, and T. Duguet, Phys. Rev. C 89, 024323 (2014).

[61] C. Barbieri and A. Carbone, Lect. Notes Phys. 936, 571 (2017).

[62] F. Raimondi and C. Barbieri, Phys. Rev. C 97, 054308 (2018).

[63] K. Tsukiyama, S. K. Bogner, and A. Schwenk, Phys. Rev. C 85, 061304(R) (2012).

[64] S. K. Bogner, H. Hergert, J. D. Holt, A. Schwenk, S. Binder, A. Calci, J. Langhammer, and R. Roth, Phys. Rev. Lett. 113, 142501 (2014).

[65] S. R. Stroberg, H. Hergert, J. D. Holt, S. K. Bogner, and A. Schwenk, Phys. Rev. C 93, 051301(R) (2016).

[66] H. Hergert, S. K. Bogner, T. D. Morris, A. Schwenk, and K. Tsukiyama, Phys. Rep. 621, 165 (2016).

[67] S. R. Stroberg, A. Calci, H. Hergert, J. D. Holt, S. K. Bogner, R. Roth, and A. Schwenk, Phys. Rev. Lett. 118, 032502 (2017).

[68] S. R. Stroberg, S. K. Bogner, H. Hergert, and J. D. Holt, Annu. Rev. Nucl. Part. Sci. 69, 307 (2019).

[69] T. Miyagi, S. R. Stroberg, J. D. Holt, and N. Shimizu, Phys. Rev. C 102, 034320 (2020).

[70] T. D. Morris, N. M. Parzuchowski, and S. K. Bogner, Phys. Rev. C 92, 034331 (2015).

[71] N. Shimizu, T. Mizusaki, Y. Utsuno, and Y. Tsunoda, Comput. Phys. Commun. 244, 372 (2019).

[72] M. Heinz, A. Tichai, J. Hoppe, K. Hebeler, and A. Schwenk, Phys. Rev. C 103, 044318 (2021).

[73] F. Coester, Nucl. Phys. 7, 421 (1958).

[74] H. Kümmel, K. H. Lührmann, and J. G. Zabolitzky, Phys. Rep. 36, 1 (1978).

[75] R. J. Bartlett and M. Musiał, Rev. Mod. Phys. 79, 291 (2007).

[76] G. Hagen, T. Papenbrock, M. Hjorth-Jensen, and D. J. Dean, Rep. Prog. Phys. 77, 096302 (2014).

[77] G. Hagen, M. Hjorth-Jensen, G. R. Jansen, R. Machleidt, and T. Papenbrock, Phys. Rev. Lett. 109, 032502 (2012).

[78] G. Hagen, G. R. Jansen, and T. Papenbrock, Phys. Rev. Lett. 117, 172501 (2016).

[79] C. G. Payne, S. Bacca, G. Hagen, W. G. Jiang, and T. Papenbrock, Phys. Rev. C 100, 061304(R) (2019).

[80] S. J. Novario, G. Hagen, G. R. Jansen, and T. Papenbrock, Phys. Rev. C 102, 051303(R) (2020).

[81] G. Hagen, T. Papenbrock, D. J. Dean, A. Schwenk, A. Nogga, M. Włoch, and P. Piecuch, Phys. Rev. C 76, 034302 (2007).

[82] R. Roth, S. Binder, K. Vobig, A. Calci, J. Langhammer, and P. Navrátil, Phys. Rev. Lett. 109, 052501 (2012).

[83] M. Bender, P.-H. Heenen, and P.-G. Reinhard, Rev. Mod. Phys. 75, 121 (2003).

[84] P. Klüpfel, P.-G. Reinhard, T. J. Bürvenich, and J. A. Maruhn, Phys. Rev. C 79, 034310 (2009).

[85] S. A. Fayans, JETP Lett. 68, 169 (1998).

[86] S. A. Fayans, S. V. Tolokonnikov, E. L. Trykov, and D. Zawischa, Nucl. Phys. A676, 49 (2000).

[87] P.-G. Reinhard and W. Nazarewicz, Phys. Rev. C 103, 054310 (2021).

[88] P.-G. Reinhard, B. Schuetrumpf, and J. Maruhn, Comput. Phys. Commun. 258, 107603 (2021). 
[89] M. Stoitsov, N. Schunck, M. Kortelainen, N. Michel, H. Nam, E. Olsen, J. Sarich, and S. Wild, Comput. Phys. Commun. 184, 1592 (2013).

[90] M. Kortelainen, J. Erler, W. Nazarewicz, N. Birge, Y. Gao, and E. Olsen, Phys. Rev. C 88, 031305(R) (2013).
[91] P.-G. Reinhard and W. Nazarewicz, Phys. Rev. C 95, 064328 (2017).

[92] R. Bollapragada, M. Menickelly, W. Nazarewicz, J. O’Neal, P.-G. Reinhard, and S. M. Wild, J. Phys. G 48, 024001 (2021). 